\title{
Review: multidisciplinary disease management programmes do not reduce death or recurrent myocardial infarction but reduce admission to hospital
}

\author{
McAlister FA, Lawson FM, Teo KK, et al. Randomised trials of secondary prevention programmes in coronary heart
} disease: systematic review. BMJ 2001 Oct 27;323:957-62.

\section{QUESTION: In patients with coronary artery disease (CAD), do multidisciplinary disease management programmes (DMPs) improve processes of care and reduce morbidity and mortality?}

\section{Data sources}

Studies were identified by searching Medline (19662000), EMBASE/Excerpta Medica (1980-99), CINAHL (1982-99), SIGLE (1980-99), the Cochrane Controlled Trials Register, and the Cochrane Effective Practice and Organization of Care Study Register using the terms case management, comprehensive health care, disease management, health services research, home care services, clinical protocols, patient care planning, quality of health care, rehabilitation, nurse led clinics, special clinics, and myocardial ischaemia. Bibliographies of identified studies were scanned and experts were contacted.

\section{Study selection}

Studies were selected if they were randomised controlled trials in any language investigating the effect of DMPs on death, myocardial infarction (MI), or patients admitted to hospital with CAD. Exclusion criteria were primary prevention studies, single modality interventions, inpatient interventions, or $<50$ patients.

\section{Data extraction}

Data were extracted by 2 independent reviewers on study quality, duration of intervention, length of follow up, and key components of the intervention.

Source of funding: Alberta Heritage Foundation for Medical Research.

For correspondence: Professor F McAlister, University of Alberta Hospital, Edmonton Alberta, Canada. Finlay.McAlister@ ualberta.ca

A modified version of this abstract appears in ACP Journal Club.

\section{Main results}

12 trials $(\mathrm{n}=9803)$ were included. Patients who received DMPs did not have greater reductions in recurrent MI (7 trials) $\{\mathrm{p}=0.44\}^{*}$ or all cause mortality (10 trials) $\{\mathrm{p}=0.40\}^{*}$ than those who received usual care (table). Admission to hospital was reduced among patients who received the intervention ( 6 trials) $\{\mathrm{p}=0.01\}^{*}$ (table). 5 of 7 trials showed reductions with DMPs in cardiovascular risk factors (cholesterol, smoking, and blood pressure). 5 of 7 trials showed increased prescriptions of $\geqslant 1$ efficacious drug (antiplatelet agents, $\beta$ blockers, or lipid lowering drugs).

Multidisciplinary disease management programme $(D M P) v$ usual care for coronary artery disease at 0.5-48 months

\begin{tabular}{lcccc} 
& \multicolumn{2}{l}{ Weighted event rates } & & \\
\cline { 2 - 4 } Outcomes & DMP & Usual care & RRR $(95 \%$ CI) & NNT (CI) \\
\hline Recurrent MI & $7.7 \%$ & $7.8 \%$ & $6 \%(-10$ to 20$)$ & Not significant \\
\hline All cause mortality & $8.04 \%$ & $8.1 \%$ & $9 \%(-4$ to 21$)$ & Not significant \\
\hline Hospital admission $\neq$ & $23 \%$ & $27 \%$ & $14 \%(4$ to 23$)$ & 30 (17 to 125$)$ \\
\hline
\end{tabular}

$\dagger \mathrm{MI}=$ myocardial infarction. Other abbreviations defined in glossary; RRR, NNT, and Cl calculated from data in article.

$\ddagger$ Calculated from data provided by author.

\section{Conclusions}

In patients with coronary artery disease, multidisciplinary disease management programmes do not reduce recurrent myocardial infarction or all cause mortality; however, admissions to hospital are reduced.

*p Values calculated from data in article

\section{COMMENTARY}

This systematic review by McAlister et al examined the effects of DMPs on processes of care, morbidity, and mortality of patients with known coronary disease. Their results indicate that DMPs do not affect short term morbidity and mortality rates, but they decrease hospital readmission rates and enhance quality of life or functional status. The relevance of reviewing these data is emphasised by the literature showing the efficacy of cardiac risk factor modification in similar patient populations. ${ }^{1}$

The strengths of this review include a thorough search of multiple databases and strict inclusion criteria. Only randomised controlled trials were included, thus, eliminating a larger pool of uncontrolled programme evaluations. The most important limitation is the broad definition of disease management used in this review: "a combination of patient education, provider use of practice guidelines, appropriate consultation, and supplies of drugs and ancillary services". Treatment programmes included in the review varied widely in both structure and process, which might challenge the appropriateness of combining these studies. Furthermore, the use of the term "disease management" leads to confusion when considering how it differs from the commonly understood concept of "cardiac rehabilitation".

Cardiac rehabilitation and secondary prevention programmes are important components of care delivery beyond acute cardiac event management. Overall, this review supports the development, implementation, and evaluation of secondary prevention programmes. Additional recommendations are made with regard to evaluating the cost effectiveness of such programmes and the assessment of long term morbidity and mortality outcomes. Finally, clarification of the terms cardiac rehabilitation, secondary prevention, and disease management is required before further advances can be made in this field of research.

Vaska Micevski, RN, ACNP, MScN Acute Care Nurse Practitioner Cardiac Program, Toronto Western Hospital University Health Network Toronto, Ontario, Canada

1 Ades PA. Cardiac rehabilitation and secondary prevention of coronary heart disease. N Engl J Med 2001;345:892-902.

2 Hunter DJ, Fairfield G. Disease management. BMJ 1997;315:50-3. 